\title{
Improving high-resolution copy number variation analysis from next generation sequencing using unique molecular identifiers
}

\author{
Pierre-Julien Viailly ${ }^{1,2^{*}}$ (D) Vincent Sater ${ }^{1,2,4 \dagger}$, Mathieu Viennot ${ }^{1,2}$, Elodie Bohers ${ }^{1,2}$, Nicolas Vergne ${ }^{5}$, \\ Caroline Berard ${ }^{4}$, Hélène Dauchel ${ }^{4}$, Thierry Lecroq ${ }^{4}$, Alison Celebi ${ }^{1,2,3}$, Philippe Ruminy ${ }^{1,2}$, Vinciane Marchand ${ }^{1,2}$, \\ Marie-Delphine Lanic ${ }^{1,2}$, Sydney Dubois ${ }^{1,2}$, Dominique Penther ${ }^{1,2}$, Hervé Tilly ${ }^{1,2}$, Sylvain Mareschal ${ }^{6}$ \\ and Fabrice Jardin ${ }^{1,2}$
}

\author{
*Correspondence: \\ pierre-julien.viailly@chb. \\ unicancer.fr \\ †Pierre-Julien Viailly and \\ Vincent Sater contributed \\ equally to this work. \\ 1 INSERM U1245, Team \\ Genomics and Biomarkers \\ of Lymphoma and Solid \\ Tumors, Normandie Univ, \\ UNIROUEN, Rouen, France \\ Full list of author information \\ is available at the end of the \\ article
}

\begin{abstract}
Background: Recently, copy number variations (CNV) impacting genes involved in oncogenic pathways have attracted an increasing attention to manage disease susceptibility. CNV is one of the most important somatic aberrations in the genome of tumor cells. Oncogene activation and tumor suppressor gene inactivation are often attributed to copy number gain/amplification or deletion, respectively, in many cancer types and stages. Recent advances in next generation sequencing protocols allow for the addition of unique molecular identifiers (UMI) to each read. Each targeted DNA fragment is labeled with a unique random nucleotide sequence added to sequencing primers. UMI are especially useful for CNV detection by making each DNA molecule in a population of reads distinct.
\end{abstract}

Results: Here, we present molecular Copy Number Alteration (mCNA), a new methodology allowing the detection of copy number changes using UMI. The algorithm is composed of four main steps: the construction of UMI count matrices, the use of control samples to construct a pseudo-reference, the computation of log-ratios, the segmentation and finally the statistical inference of abnormal segmented breaks. We demonstrate the success of mCNA on a dataset of patients suffering from Diffuse Large B-cell Lymphoma and we highlight that $\mathrm{mCNA}$ results have a strong correlation with comparative genomic hybridization.

Conclusion: We provide mCNA, a new approach for CNV detection, freely available at https://gitlab.com/pierrejulien.viailly/mcna/ under MIT license. mCNA can significantly improve detection accuracy of CNV changes by using UMI.

Keywords: UMI, CNV calling, Next generation sequencing

\section{Background}

Recently, copy number variations (CNV) impacting genes involved in oncogenic pathways have attracted an increasing attention to manage disease susceptibility $[1,2]$. $\mathrm{CNV}$ is one of the most important somatic aberrations in the genome of tumor cells. author(s) and the source, provide a link to the Creative Commons licence, and indicate if changes were made. The images or other third party material in this article are included in the article's Creative Commons licence, unless indicated otherwise in a credit line to the material. If material is not included in the article's Creative Commons licence and your intended use is not permitted by statutory regulation or exceeds the permitted use, you will need to obtain permission directly from the copyright holder. To view a copy of this licence, visit http:// creativecommons.org/licenses/by/4.0/. The Creative Commons Public Domain Dedication waiver (http://creativecommons.org/publi cdomain/zero/1.0/) applies to the data made available in this article, unless otherwise stated in a credit line to the data. 
Oncogene activation and tumor suppressor gene inactivation are often attributed to copy number gain/amplification or deletion, respectively, in many cancer types and stages.

$\mathrm{CNV}$ analysis refers to the detection of a difference in the dosage of a genomic locus containing one or more dosage-sensitive genes (zygosity). The resolution limit of conventional cytogenetics (approximately $5 \mathrm{Mb}$ ) has been improved by molecular cytogenetics using comparative genomic hybridization $(\mathrm{CGH})$ and more recently array comparative genomic hybridization (aCGH). These technologies make it possible to detect genomic imbalances of $<100 \mathrm{~kb}$, whereas more specialized array designs increase the resolution to $\leq 200 \mathrm{bp}$ for specific targeted regions. Despite these performances, aCGH requires the purchase of a specific platform for data acquisition and its resolution is limited to the detection of tumoral clones that differ substantially in DNA content from a reference.

Next Generation Sequencing technologies (NGS) have rapidly supplanted traditional Sanger sequencing as the preferred methodology for the detection of actionable single nucleotide variations (SNV) in oncology. Diagnostic laboratories are now massively equipped with Illumina/Thermofisher sequencers. Massively parallel sequencing offers many advantages including high sensitivity and specificity for SNV and CNV detection within a single platform. Nevertheless, libraries must be amplified by PCR to produce a sufficient amount of signal. This amplification step introduces many biases for counting reads because the number of produced reads is no longer directly proportional to the number of initial unique targeted DNA fragments. The amplification factor of each region is unknown and depends on many parameters such as library size, GC content, region length or competition between primers overlapping the same locus while using amplicon-based libraries.

There are three main approaches to identify CNV from NGS data: read-pair (RP), split-read (SR), and read-depth (RD).

RP methods (BreakDancer [3], PEMer [4], Ulysses [5]) consist in comparing the average insert size between the sequenced read-pairs with an expected size based on a reference genome. The discordance between mapped paired-reads and the predetermined average insert size is then used to identify gain and loss of materials. Shorter/longer insert size than expected will correlate to the loss/gain of material, respectively.

SR methods evaluate CNV using paired reads where only one read of the pair has a reliable mapping quality whereas the other one partially fails to map to the reference sequence. These discrepancies within a read pair can potentially provide the precise position of insertion/deletion events. Several tools implementing SR strategies enable the detection of these breakpoints (SVseq2 [6], Gustaf [7], PRISM [8]) but they are limited to short insertions or deletions.

The RD approach consists in counting aligned reads overlapping a genomic region in a sliding window. These read counts $(\mathrm{RC})$ are then compared between the sample of interest and a reference to compute $\mathrm{CNV}$ segmentation. A local decrease in sequencing depth will be associated with a loss of genomic material whereas its increase will be correlated to locus gain/amplification. Several tools were developed using RD-based approaches (CNVnator [9], CNV-seq [10]). This strategy seems particularly promising for the analysis of targeted sequencing experiments (TSE). TSE enables the sequencing of key genes or regions of interest to high depth (500-1000X or higher) and provides a cost-effective 
strategy to identify variants at low allele frequencies. Some tools, such as ONCOCNV [11], were specially developed for the analysis of targeted amplicon-based libraries. Many biases due to the amplification step while preparing this type of library prevent the direct quantification of loci copy-number (size of the library, GC percentage, amplicon length, primer melting temperature, competition between primers...). It implies the use of normalization strategies to allow the comparison of read counts between samples.

Recent advances in NGS protocols allow for the addition of unique molecular identifiers (UMI) to each read. Each targeted DNA fragment is labeled by a unique random nucleotide sequence added to sequencing primers. UMI are especially useful for CNV detection by making each DNA molecule in a population of reads distinct. They allow the direct count of targeted DNA molecules before the library amplification by simply counting the number of unique UMI sequences per position of the alignment.

Here, we present mCNA (molecular Copy Number Alteration), a new methodology allowing the detection of copy number changes using UMI. We demonstrate the success of our algorithm on a dataset of patients diagnosed with Diffuse Large B-cell Lymphoma (DLBCL) and we highlight that mCNA results have a strong correlation with CGH. To assess the robustness and sensitivity limit of our approach, we used in silico simulation of copy number aberrations in a control sample and also sequential dilutions of REC-1 cell line.

\section{Methods}

\section{Library construction}

A Pan-lymphoma panel was designed using the QIAseq Targeted DNA Custom Panel Builder (QIAGEN) to identify alterations within important genes for lymphomagenesis. This panel targets 69 genes (hotspots, regions or whole gene) using 1493 gene specific primers. List of genes and number of GSP per gene are provided in Additional file 1: Table S1.

The QIAseq Targeted DNA chemistry introduces molecular barcodes (UMI) to enable digital sequencing and to identify PCR duplicates (Fig. 1). The molecular barcodes are short aleatory nucleotide sequences of 12 bp length added to each read before the library amplification. Statistically, this process provides $4^{12}$ possible indices per adapter; hence, each DNA molecule in the sample receives a unique UMI sequence.

\section{Subjects and methods}

\section{Study design and patients}

22 adult patients with de novo CD20+ Diffuse Large B-cell Lymphoma (DLBCL) or primary mediastinal B-cell lymphoma (PMBL) were selected from the prospective, multicenter, and randomized LNH-03B LYSA trials with available frozen tumor samples and adequate DNA quality. CGH was previously performed for these samples after wholegenome amplification against a Promega normal DNA pool using Agilent SurePrint G3 $4 \times 180 \mathrm{~K}$ microarrays. Briefly, arrays were scanned with Agilent Feature Extraction and processed with cghRA pipeline as previously described [12].

DNA from REC-1 cell line, established from the lymph node of a 61-year-old man with refractory B-cell lymphoma, was extracted. Dilutions at 50\%, 30\%, 20\%, $10 \%$ and 


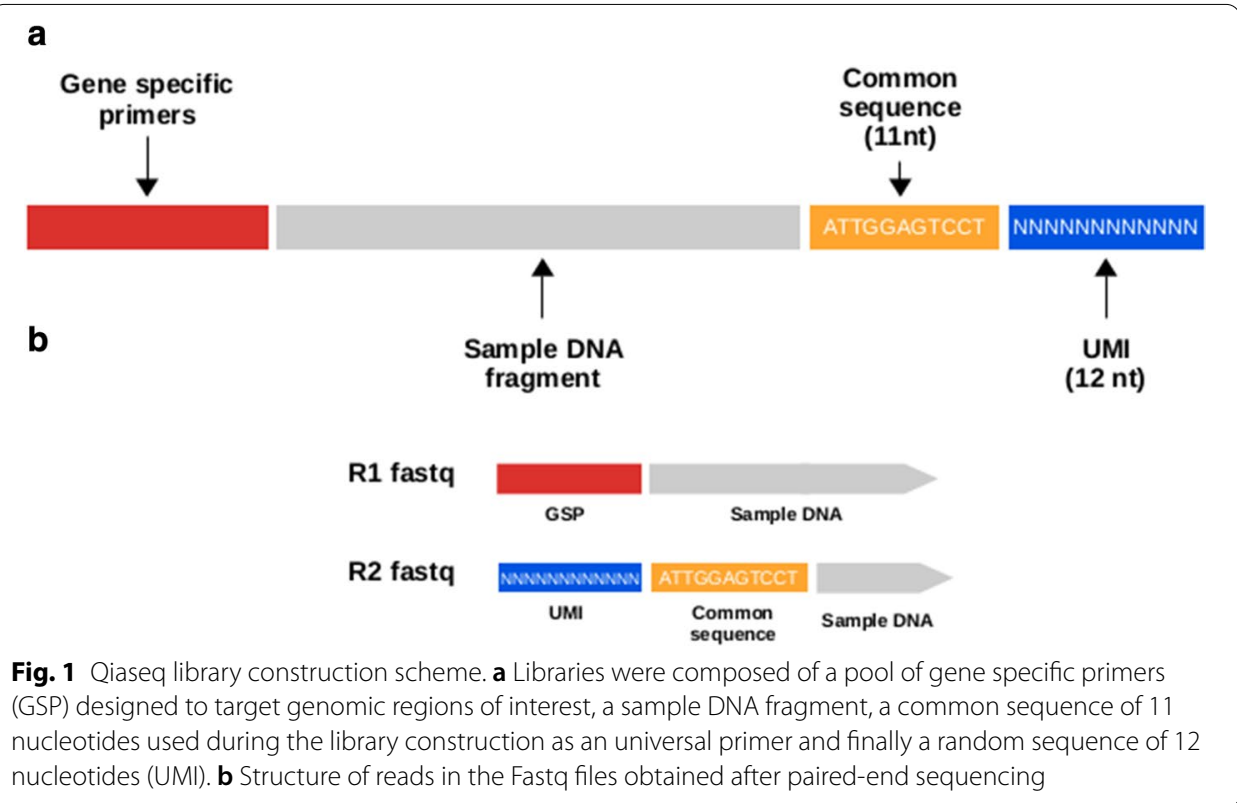

$5 \%$ of this DNA were performed using Human Mixed Genomic DNA Promega. Human Genomic DNA comes from multiple anonymous donors.

Five blood samples of healthy individuals were collected and used as a control to construct the pseudo-reference profile.

\section{Sample collection and sequencing}

Tumor genomic DNA (gDNA) was isolated from fresh diagnostic tissue biopsies or blood. Samples were quantified using QuBit High Sensitivity dsDNA (Thermo Fisher Scientific).

gDNA samples were sequenced with the entire Pan-lymphoma panel. 30 ng of gDNA were enzymatically fragmented and end repaired, followed by ligation of the molecular barcoded adaptators (UMI). After purification, target enrichment was carried out using the set of 1493 gene specific primers. Then, enriched DNA was submitted to universal PCR with a number of cycle adapted to this number of primers. Purified libraries were quantified using QuBit High Sensitivity dsDNA.

Finally, libraries were sequenced on Illumina MiSeq (paired-end, $2 \times 150 \mathrm{bp}$ ) following manufacturer's user manual (Illumina, CA).

\section{Library sequencing and bioinformatics pre-processing}

Briefly, gene-specific primers and common regions were trimmed from R1 and R2 fastq using an in-house program. UMI sequences were extracted from read construction using UMI-tools [13].

Reads were aligned against hg19 reference genome using BWA-mem [14] and standardized according to the GATK3 Best Practices recommendations. A detailed bioinformatics pipeline is provided in Additional file 1: Fig. S1. 


\section{mCNA algorithm}

In this article, we present a new strategy to detect copy number changes for targeted panels of genes using UMI. The algorithm is composed of four main steps: the construction of UMI count matrices, the use of control samples to construct a pseudo-reference, the computation of log-ratios (LR), the segmentation and finally the statistical inference of abnormal segmented breaks (Fig. 2).

\section{Prerequisites}

mCNA algorithm requires sequencing libraries introducing one or more short aleatory sequences (Unique Molecular Identifiers, UMI) in reads construction. UMI sequences

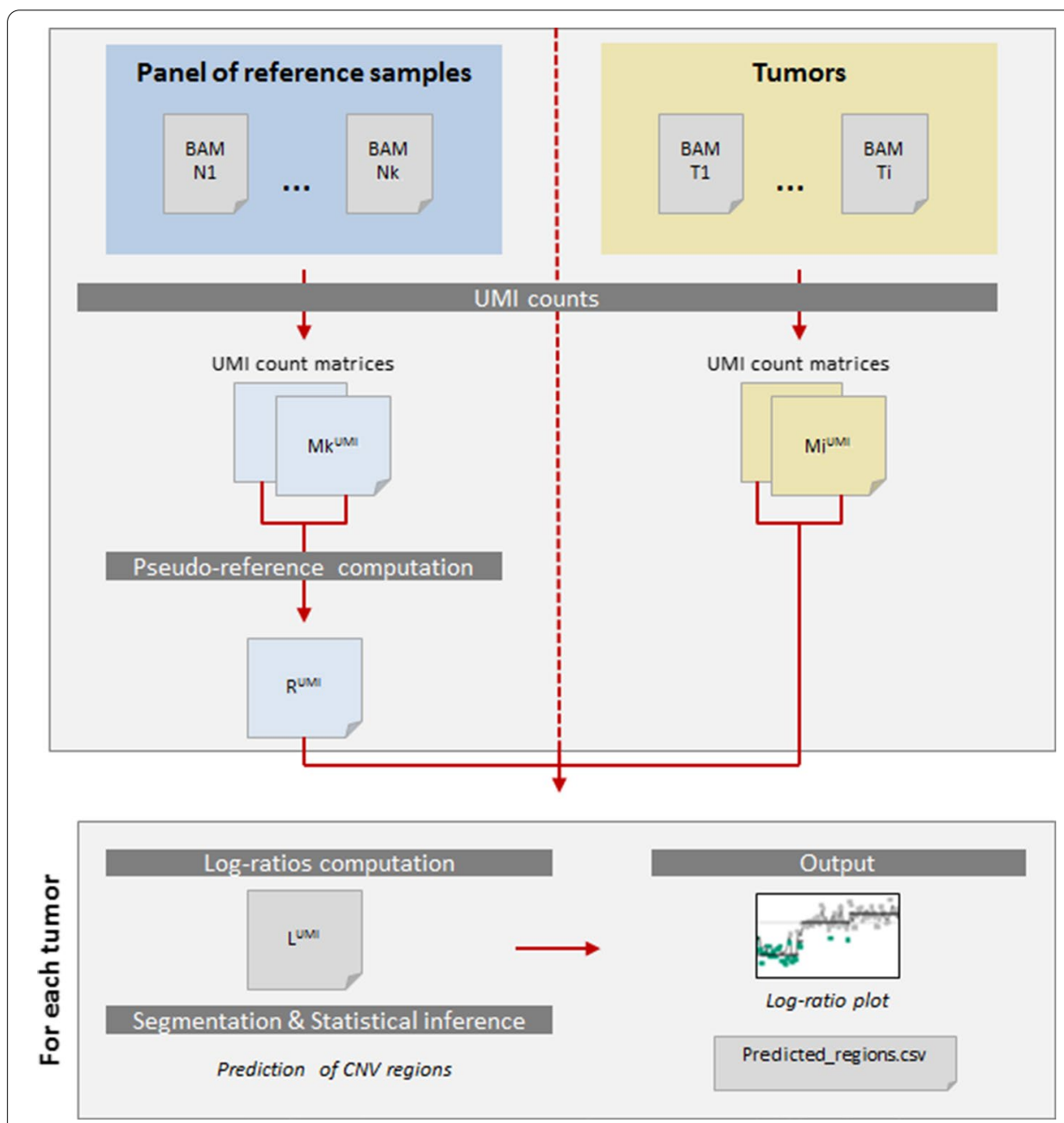

Fig. 2 Workflow of mCNA data processing. Processing of control samples: Matrices of UMI counts are constructed by counting number of UMI overlapping each targeted region from the BED file. Then, each value of both matrices are divided respectively by the mean UMI-depth to standardize sample counts. A pseudo-reference is constructed giving for each region the geometric mean of observed UMI-depth. Processing of tumoral samples: Using the same processes than for reference samples, UMI-depth matrices are computed and normalized. Log-ratios computing and segmentation: log-ratios are computed and segmentation is then performed for each gene by CBS. Statistical tests are performed to determine whether observed signals within segments are significantly distinct from 0 
must be extracted from raw FASTQ files before alignment and appended to read identifiers using UMI-tools [13]. Processed reads must be aligned against a reference genome to produce BAM file. A BED file is also required, giving for each targeted region the chromosome name, the start/end positions of the locus and the gene name.

Details for complete bioinformatics processing of QIAseq Targeted DNA Panel are provided in Additional file 1: Fig. S1.

\section{Construction of UMI-depth matrices}

We define $M^{\mathrm{UMI}}$ as the UMI-depth matrix of one BAM file. $P$ is the total number of targeted regions. $C_{\mathrm{p}}^{\mathrm{UMI}}$ reflects the number of unique $\mathrm{UMI}$ overlapping $p$ region and $U$ the total number of unique UMI of one sample.

Each region supplied in the BED file is scanned using scanBam function of Rsamtools package [15]. $C_{\mathrm{p}}^{\mathrm{UMI}}$ is computed from unique UMI sequences extracted from read names overlapping $p$.

Each matrix $M^{\mathrm{UMI}}$ is finally normalized by $U$ to allow the comparison between samples, as shown in the Additional file 1: Fig S2.

\section{Pseudo-reference construction}

From $M^{\mathrm{UMI}}$ matrices of normal samples, a geometric mean of $C_{\mathrm{p}}^{\mathrm{UMI}} / U$ is computed line by line to create a vector $R^{\mathrm{UMI}}$ of dimensions $(1, P)$.

To automatically detect outlier samples, Root-Mean-Square Deviations (RMSD) are computed between $C_{\mathrm{p}}^{\mathrm{UMI}} / U$ and $R_{\mathrm{p}}^{\mathrm{UMI}}$ for each region p of each control sample.

Samples with at least $20 \%$ of regions with $R M S D_{p}>T$ are excluded from baseline construction, with $T$ defined as:

$$
T=Q 3\left(R M S D_{p}\right)+1.5 \times I Q R\left(R M S D_{p}\right)
$$

If at least one sample is filtered, $R^{\mathrm{UMI}}$ vector is updated with passing filter $M^{\mathrm{UMI}}$ matrices only. The same process is applied to detect outlier noisy regions. These positions are defined as sequenced regions with at least $R M S D_{p}>T$ in $50 \%$ of control samples.

\section{Log-ratios and signal centering}

We define the $\log$-ratio $L_{\mathrm{p}}^{\mathrm{UMI}}$ as:

$$
L_{\mathrm{p}}^{\mathrm{UMI}}=\log 2\left(\frac{M_{\mathrm{p}}^{\mathrm{UMI}}}{R_{\mathrm{p}}^{\mathrm{UMI}}}\right)
$$

where $M_{\mathrm{p}}^{\mathrm{UMI}}$ is the UMI count of a tumor sample for the region $p$ and $R_{\mathrm{p}}^{\mathrm{UMI}}$ is the UMI pseudo-reference vector of control samples for the region $p$.

A Gaussian mixture model with one to three mixture components is estimated from $L_{\mathrm{p}}^{\mathrm{UMI}}$ using Mclust function of R package mclust [16]. The estimated gaussian closest to $\widehat{L^{\mathrm{UMI}}}=0$ is used to center the signal by subtracting its average from the $L_{\mathrm{p}}^{\mathrm{UMI}}$ values. Indeed, we assume that the Gaussian of our signal closest to 0 corresponds to a diploid state. This centering step could be disabled via the program's arguments. 


\section{Segmentation}

Each gene is composed of $n$ consecutive regions and we define a vector of $\log$-ratios $V_{\mathrm{n}}^{\mathrm{UMI}}$ used for segmentation, as:

$$
V_{\mathrm{n}}^{\mathrm{UMI}}=\left\{L_{\mathrm{p}}^{\mathrm{UMI}} ; L_{\mathrm{p}+1}^{\mathrm{UMI}} ; \cdots\right\}(p \in n)
$$

mCNA uses the circular binary segmentation (CBS) method implemented in the R package PSCBS [17] to segment $V_{\mathrm{n}}^{\mathrm{UMI}}$.

To avoid breakpoints at outlier values, a vector of weights $W$ is given to CBS segmentation function. $W$ is inversely proportional to the variances of $C_{\mathrm{p}}^{\mathrm{UMI}} / U$ observed in the control samples and defined as:

$$
W_{\mathrm{p}}=\frac{1}{\operatorname{var}\left(M_{\mathrm{p}}^{\mathrm{UMI}} / U\right)}(\text { within controls })
$$

$W_{\mathrm{p}}$ are then transformed to be limited to the interval $[0,1]$ as follows:

$$
W_{\mathrm{p}}^{\prime}=\frac{W_{\mathrm{p}}-\min \left(W_{\mathrm{p}}\right)}{\max \left(W_{\mathrm{p}}\right)-\min \left(W_{\mathrm{p}}\right)}
$$

Finally, a Student's t-test is performed on each segmented region to test whether or not the $V_{\mathrm{n}}^{\mathrm{UMI}}$ vector is significantly different from the reference value of 0 . To avoid false positive segments, a FDR correction is applied.

\section{Estimation of tumoral content}

We define $G_{\mathrm{n}}^{\mathrm{UMI}}$ and $D_{\mathrm{n}}^{\mathrm{UMI}}$ the vectors $V_{\mathrm{n}}^{\mathrm{UMI}}$ of significant amplified/deleted segments, respectively. We use $D_{\mathrm{n}}^{\mathrm{UMI}}$ and $G_{\mathrm{n}}^{\mathrm{UMI}}$ distributions to estimate tumor enrichment assuming that means of these distributions reflect a gain/loss of one segment copy and that log-ratios are a mixture of both tumoral and normal signals. We define as $c$ the percentage of tumor enrichment to estimate.

Two independent estimates of $c$ were produced: one from the significantly deleted segments and the other from those amplified. The estimation of $c$ cannot be done in one step because log-ratios involving one gain or one loss are not symmetrical. For example, the loss of one copy of a segment in a sample containing only tumor cells will lead to a log-ratio equal to $\log _{2}\left(\frac{1}{2}\right)=-1$ while a gain of one copy will lead to $\log _{2}\left(\frac{3}{2}\right)=0.58$.

From amplified regions, the distribution of $L_{\mathrm{p}}^{\mathrm{UMI}}$ can be decomposed as follows:

$$
\begin{aligned}
L_{\mathrm{p}}^{\mathrm{UMI}}=\log _{2}\left(c \times \frac{3}{2}+(1-c) \times \frac{2}{2}\right) & \Longleftrightarrow 2^{L_{\mathrm{p}}^{\mathrm{UMI}}}=\frac{1}{2} c+1 \\
& \Longleftrightarrow c=2\left(2^{L_{\mathrm{p}}^{\mathrm{UMI}}}-1\right)
\end{aligned}
$$

The mean value of the distribution of $G_{\mathrm{n}}^{\mathrm{UMI}}$ is used in order to complete this Eq. (1) to estimate $c$.

The same decomposition is carried out considering the loss of a copy:

$$
L_{\mathrm{p}}^{\mathrm{UMI}}=\log _{2}\left(c \times \frac{1}{2}+(1-c) \times \frac{2}{2}\right) \Longleftrightarrow c=-2\left(2^{L_{\mathrm{p}}^{\mathrm{UMI}}}-1\right)
$$


The mean value of the distribution of $D_{\mathrm{n}}^{\mathrm{UMI}}$ is used in order to complete this Eq. (2) to estimate $c$.

The algorithm output by default the mean value of this two independant estimates of $c$

\section{Results}

\section{Comparison between read-depth and UMI-depth signals}

To allow the comparison between read-depth and UMI-depth signals, we extracted respective counts from our reference samples for each targeted region. Theses counts were normalized respectively by the mean read-depth/the mean UMI-depth to make samples comparable. Measured variances were significantly lower when taking into account the UMI-depth and not the read-depth ( $p$ value $<2.2 \mathrm{e}-16$ ), as shown in Fig. 3.

\section{Construction of Pan-Lymphoma baseline}

From mCNA quality control step, one control sample (CTL-22081) was excluded during pseudo-reference computation because of too high RMSD. The distribution of normalized UMI counts for this sample was clearly distinct from others as shown in the

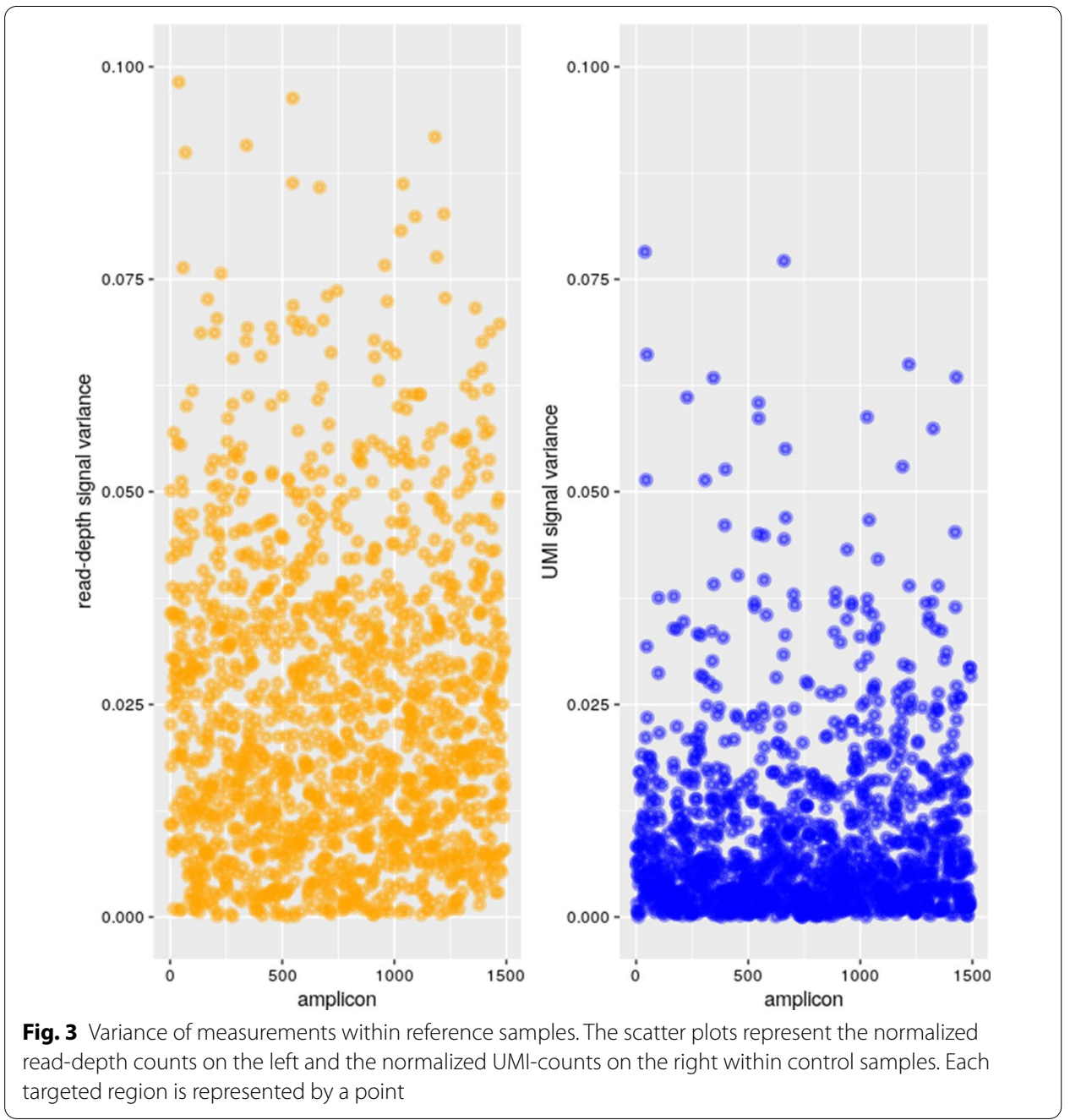


Additional file 1: Fig. S2. mCNA also detected 31 targeted regions not passing RMSD filters which were excluded. List of outliers and their characteristics are provided in Additional file 1: Table S2.

To validate our approach, we determined the correlation between normalized UMI count matrices of control samples and the computed pseudo-reference vector for each targeted region (Fig. 4). Signals were significantly and strongly correlated ( $r>0.96$, $p<2.2 \mathrm{e}-16)$, which means that the computed pseudo-reference perfectly reflects the controls.

\section{Example of mCNA profile}

For each tested sample, mCNA generates a csv file summarizing by segment the measured data and the significance of the tests. A graph is also provided representing the logratios by region, the segmented signal and the results of the test. An example of profile is shown in Fig. 5.

\section{Comparison between mCNA and CGH data}

In order to validate mCNA approach, we first compared CGH and NGS data (Fig. 6). We estimated log-ratios for each targeted region of the Pan-lymphoma panel using mCNA approach and then those estimated from CGH.

We observe a strong correlation between log-ratios of both technologies $(r=0.74)$. The majority of discrepancies are visible for $L_{\mathrm{CGH}}=0$ which may show a lack of sensitivity of CGH due to a lack of probe coverage.

To further our comparison, we extracted all predicted mCNA segments of our 22 tumor samples. These segments were then annotated with CGH results. 114/120 (95\%)

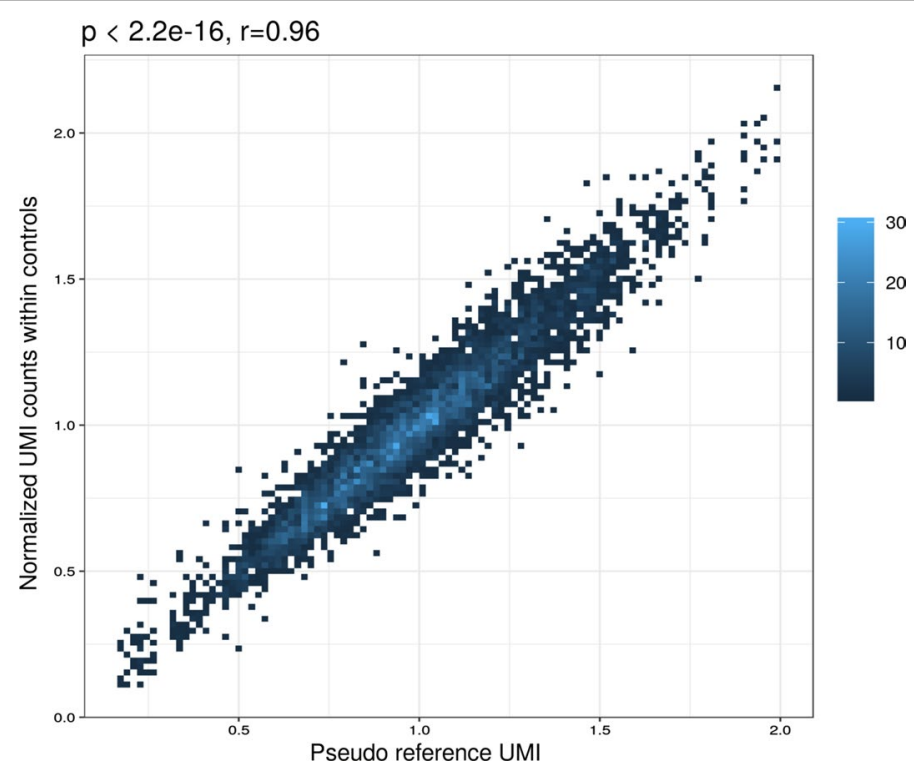

Fig. 4 Correlation between normalized UMI count matrices and matched pseudo-reference vector. The scatter plot represents the normalized counts $C_{p}^{U M I} / U$ of $M^{U M I}$ matrices within control samples and the associated pseudo-reference vector $R^{\mathrm{UMI}}$. Each targeted region is represented by a point. A Pearson correlation test is computed and correlation coefficient is displayed at the top of the figure 


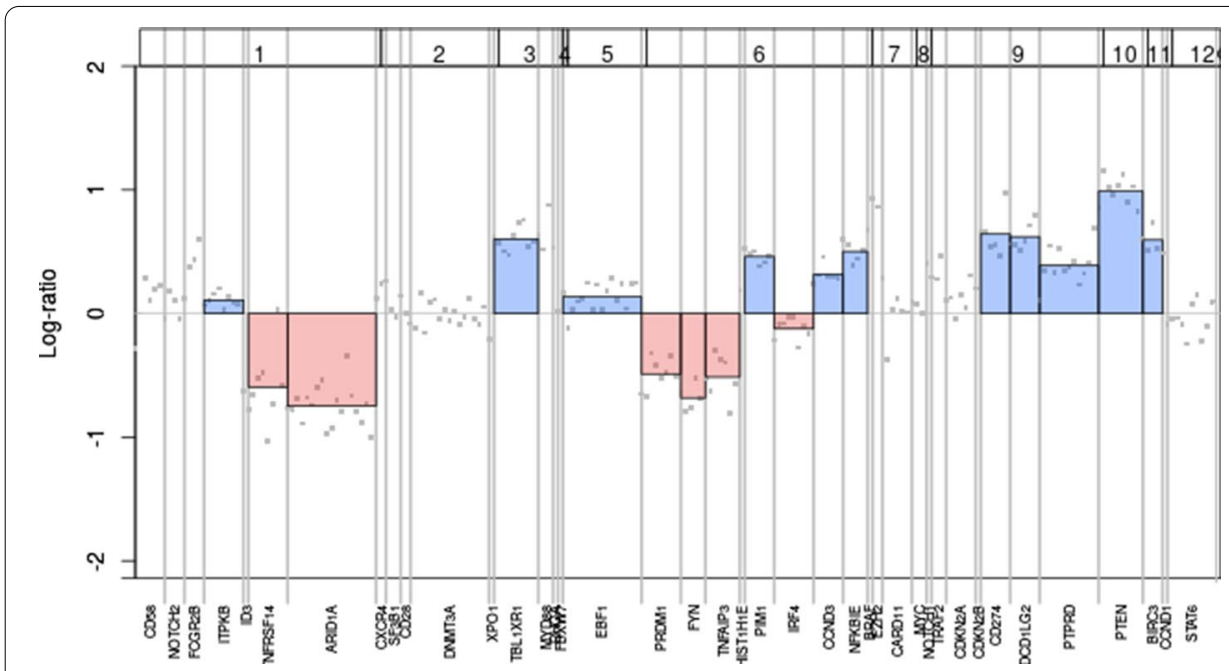

Fig. 5 Example of mCNA profile. The top of the panel represents the chromosome location. Each vertical line delimits a gene. The log-ratio plot gives estimated log-ratios for the sample and the results of CBS segmentation. Blue/red segments represent respectively significant gain/lost segments. Gene names are displayed at the bottom of the graph

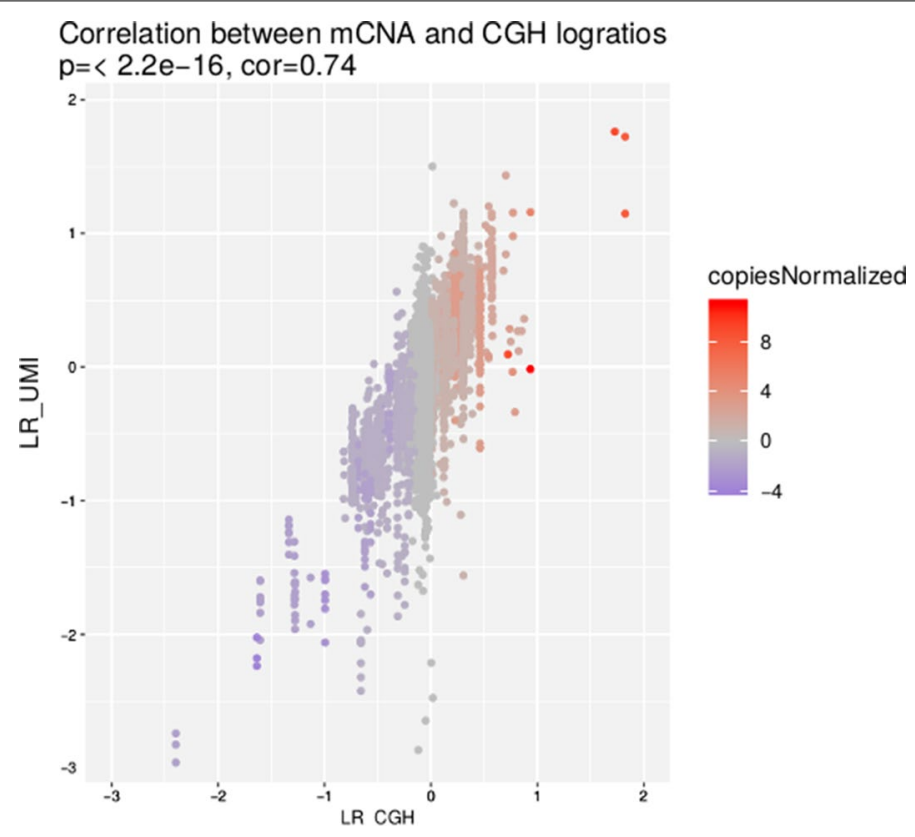

Fig. 6 Correlation between $\mathrm{mCNA}$ and $\mathrm{CGH}$ log-ratios. Each point of the plot corresponds to a region of the Pan-Lymphoma Panel annotated by CGH results and estimated by mCNA. Colors show the number of gain or loss estimated by CGH data regarding the region. Pearson correlation coefficient is displayed at the top of the plot

mCNA segments were predicted deleted by CGH and 175/221 (79\%) were predicted as gain. 723/978 were predicted normal by mCNA and confirmed in CGH (74\%), leading to an overall agreement between the two datasets of $83 \%$. 


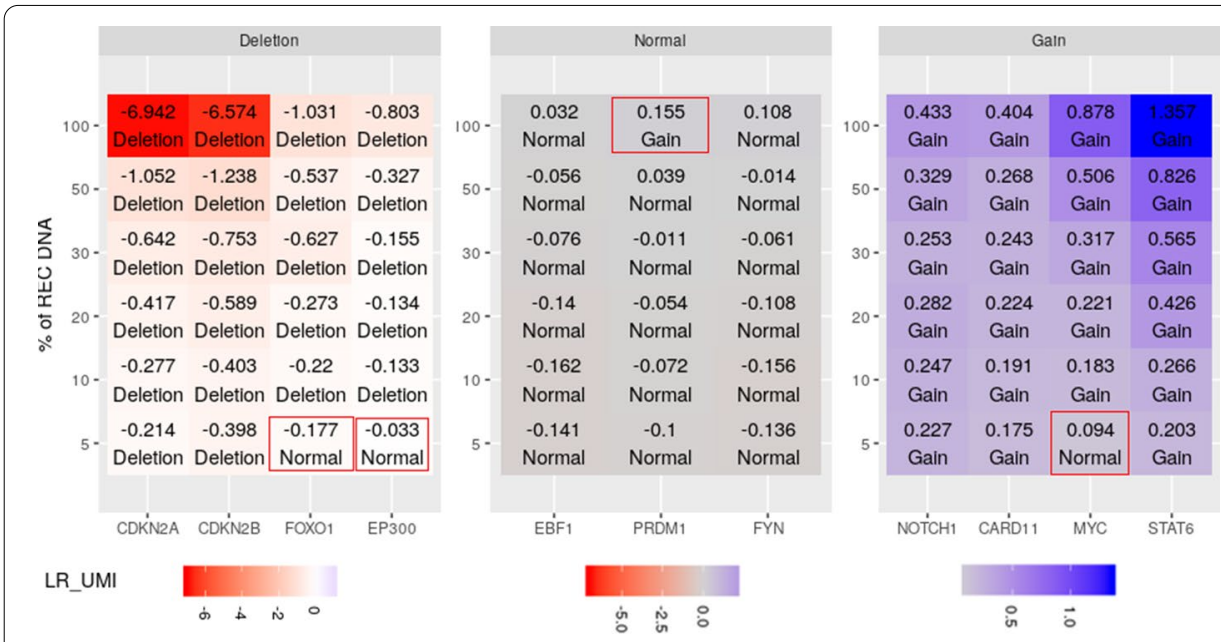

Fig. 7 mCNA results of REC-1 cell line through dilutions. Each cell contains the log values obtained for the given gene and the final status of the segment given by mCNA

\section{Robustness and sensitivity limit}

To estimate theoretical sensitivity limit of mCNA approach, we first edited $M^{\mathrm{UMI}}$ matrix of UMI count of one control sample $(16,464)$ to introduce amplification of XPO1, gain of IRF4, heterozygous deletion of CDKN2A and homozygous deletion of CDKN2B. We applied an in silico dilution of these abnormal segments at 100\%, 50\%, 20\%, 10\% and 5\% of tumor cells and applied mCNA to determine whether or not segments were significantly found after signal centering, segmentation and statistical test application. Results were summarized in Additional file 1: Fig. S4 and Additional file 1: Table S3. We found a strong correlation between expected and computed log-ratios $(\mathrm{r}=0.99, p=7.19 \mathrm{e} 8-27)$ after signal centering and segmentation. mCNA was able to detect all in silico abnormal segments for tumor cell percentage between $10 \%$ and $100 \%$. At $5 \%$, only segments involving gain or loss of more than one copy were significantly found.

To confirm in silico results, REC-1 cell line was sequenced on two different runs to estimate the robustness of $L_{\mathrm{p}}^{\mathrm{UMI}}$ measurement using the Pan-Lymphoma panel. We found a strong correlation between the two replicates $(\mathrm{r}=0.93, p<0.001)$ even if the sequencing depths were not the same (1851X/2217X). Details are provided in Additional file 1: Fig. S3.

30/31 segments were predicted as gains in both replicates (96.77\%), 21/23 (91.30\%) as normal and 17/18 (94.44\%) as deleted, thus giving an average agreement of $94.17 \%$. Discordant predictions result from segments having a low number of targeted regions and a small log-ratio variation.

Finally, dilutions of REC-1 DNA were performed at 50\%,30\%, 20\%, $10 \%$ and $5 \%$. REC-1 is a near-diploid cell line of male origin with a modal chromosome number of 45 and a polyploidy rate of $10 \%$. Its karyotype is highly rearranged with approximately 5-6 derivative chromosomes in the karyology that have been described. Significant segments in this cell line were selected from the initial profile to evaluate the sensitivity of our approach through the different dilutions. Results seem consistent up to a threshold of $10 \%$ enrichment (Fig. 7). Above this threshold, the evaluation of tumor content 


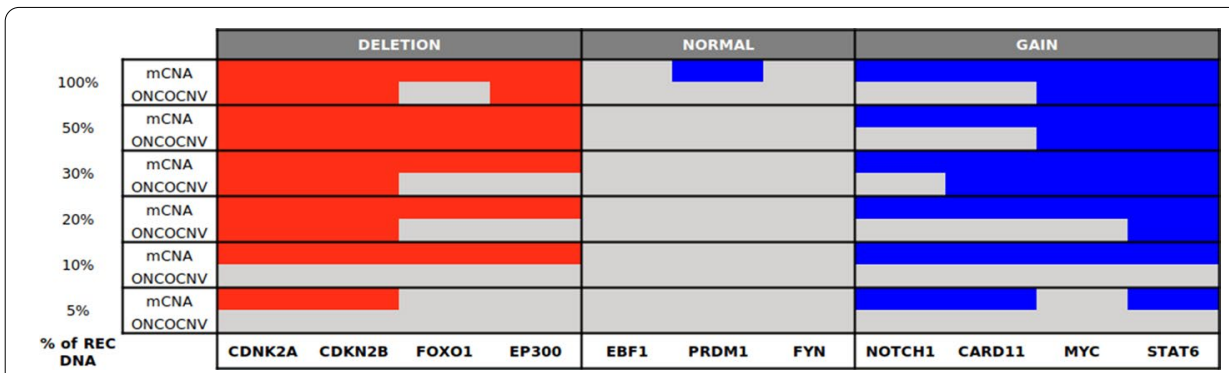

Fig. $8 \mathrm{mCNA}$ and ONCOCNV results of REC-1 cell line through dilutions. Each cell contains the calls obtained for the given gene

seems consistent between expected and estimated percentage of tumor cells $(r=0.98)$ as shown in Additional file 1: Fig. S5.

\section{Comparison to read-depth algorithm}

To assess mCNA's analytical performance, we decide to compare our UMI-depth approach to the read-depth algorithm ONCOCNV [11] using REC-1 dataset. ONCOCNV was commonly used for the analysis of targeted sequencing panel of genes. It uses several normalization steps on read counts to erase library amplification biases such as library size, GC content of each region or amplicon length.

We hypothesized that the direct count of UMI could improve the limit sensitivity of ONCOCNV insofar as we have shown that the signal in UMI was less noisy than read counts. ONCOCNV results were generated for all REC-1 dilutions using the same control samples as those used to construct mCNA baseline.

As expected, mCNA achieved much higher prediction accuracies than ONCOCNV as the percentage of tumor cells decreases (Fig. 8). Here, accuracy measures the proportion of genes with correctly annotated copy number status compared to the initial REC-1 profile: normal, gain or deletion. Considering the results of the algorithms from 100 to $10 \%$ of REC-1 DNA, the overall prediction accuracy fluctuated from 0.90 to 0.27 for ONCOCNV, while it was significantly higher for mCNA : 1.0 to 0.90 . Interestingly, while mCNA results look consistent at $10 \%$, ONCOCNV fails to detect heterozygous deletions of $F O X O 1$ and EP300 at 30\% of tumoral cell.

\section{Discussion}

We proposed a new methodology to be used to detect copy number changes for targeted panels of genes using unique molecular identifiers. By changing the source of information from sequencing depth to UMI depth, mCNA provides a simple and robust methodology for the detection of CNV.

We demonstrated that using UMI-depth signal, and not read-depth signal, seems more robust in samples without abnormal copies. The algorithm uses a pool of reference samples to construct a pseudo-reference and includes a filtering step to automatically exclude samples and/or targeted regions with abnormal variance. We 
demonstrated that this in silico baseline profile reflects the reference samples and enables the estimation of $\mathrm{CNV}$ changes in unpaired tumor samples.

mCNA provides a strong estimation of log-ratios which correlates to our CGH dataset of 22 DLCBL samples. As we expected, the majority of discrepancies are visible for short breaks within genes probably due to a lack of probe coverage of Agilent SurePrint G3 4x180K microarrays. To avoid overestimation of breakpoints due to outlier values, mCNA provides a vector of weights to CBS segmentation function. We also recommend the use of at least 6 non-overlapping amplicons to properly estimate the state of a targeted region.

As we expected, we failed to detect CNA for samples that were highly contaminated by normal cells (less than $10 \%$ of tumor content). In this case, the noise in measurements is higher than the expected difference between measurements in the case of one CNV event. This observed threshold of $10 \%$ was confirmed by in silico simulation and also by sequential dilution of REC- 1 cell line.

Our approach is designed to be used for targeted gene panels and thus doesn't allow the combination of UMI-depth signal and B allele frequencies to improve the sensitivity of our CNV calling approach, as for analyses at the exome scale for example.

Another limitation of mCNA approach is the assumption that the majority of the signal corresponds to a diploid state. Polyploid profiles for example still remain challenging because the algorithm proceeds to center the signals. We recommend for panels targeting very frequently altered genes to deactivate this centering step.

Finally, mCNA gives the opportunity to obtain both the mutational and the copy number status at no additional cost. It helps in the interpretation of frequently altered genes, such as TP53 for example, for which mutations are often associated with copy abnormalities.

\section{Conclusion}

In this article, we present a new strategy to detect copy number changes for targeted panels of genes using UMI. mCNA is composed of four main steps: the construction of UMI count matrices, the use of reference samples to construct a pseudo-reference, the computation of log-ratios, the segmentation and finally the statistical inference of segmented breaks.

\section{Abbreviations}

CNV: Copy number variation; UMI: Unique Molecular Identifiers; mCNA: Molecular Copy Number Alteration; LR: Log-ratio; NGS: Next Generation Sequencing; SNV: Single Nucleotide Variation; PCR: Polymerase chain reaction; RP: Read-pair; SR: Split-read; RD: Read-depth; RC: Read count; TSE: Targeted Sequencing Experiment; DLBCL: Diffuse Large B-Cell Lymphoma; PMBL: Primary Mediastinal B-cell Lymphoma; GSP: Gene Specific Primer; BED: Browser Extensible Data; BAM: Binary Alignment Map; RMSD: Root-Mean-Square Deviation; FDR: False Discovery Rate.

\section{Supplementary Information}

The online version contains supplementary material available at https://doi.org/10.1186/s12859-021-04060-4.

Additional file 1. Supplementary Figures S1-S5, Supplementary Tables S1-S3. 


\section{Authors' contributions}

PJV and VS conceived the algorithm. MV, MDL and EB performed the experiments. NV, CB, HD, TL, AC and SM contributed to the statistical design of the study. PR, VM, MDL, DP, and HT contributed to data interpretation. PJV, VS, SD and FJ contributed to the writing of the manuscript. All authors discussed the results and contributed to the final manuscript. All authors read and approved the final manuscript.

\section{Funding}

This study was funded by grants from the Centre Henri Becquerel (Rouen, France). The funder had no role in study design, data collection and analysis, decision to publish, or preparation of the manuscript.

\section{Availability of data and materials}

mCNA is available at https://gitlab.com/pierrejulien.viailly/mcna/ under MIT license. The datasets analysed during the current study are also available in mCNA data repository.

\section{Declarations}

\section{Ethics approval and consent to participate}

Sequencing data results from patients enrolled in the prospective, multicenter, and randomized LNH-03B LYSA clinical trials. This study was performed with approval of the Ethic Committee Haute-Normandie on 2003 and written informed consent was obtained from all participants at the time of enrollment.

\section{Consent for publication}

Not applicable.

\section{Competing interests}

The authors declare that they have no competing interests.

\section{Author details}

' INSERM U1245, Team Genomics and Biomarkers of Lymphoma and Solid Tumors, Normandie Univ, UNIROUEN, Rouen, France. ${ }^{2}$ Centre Henri Becquerel, Rouen, France. ${ }^{3}$ Master Bioinformatique BIM, Normandie Univ, UNIROUEN, Rouen, France. ${ }^{4}$ LITIS EA 4108, Normandie Univ, UNIROUEN, Rouen, France. ${ }^{5}$ LMRS UMRS 6085, Normandie Univ, UNIROUEN, Rouen, France. ${ }^{6}$ INSERM U1052 UMR CNRS 5286, Cancer Research Center of Lyon, Lyon, France.

Received: 10 August 2020 Accepted: 2 March 2021

Published online: 12 March 2021

\section{References}

1. Shlien A, Malkin D. Copy number variations and cancer. Genome Med. 2009;1 (6):62.

2. Jardin F, Jais J-P, Molina T-J, Parmentier F, Picquenot J-M, Ruminy P, Tilly H, Bastard C, Salles G-A, Feugier P, Thieblemont C, Gisselbrecht C, de Reynies A, Coiffier B, Haioun C, Leroy K. Diffuse large B-cell lymphomas with CDKN2a deletion have a distinct gene expression signature and a poor prognosis under R-CHOP treatment: a GELA study. Blood. 2010;116(7):1092-104.

3. Fan X, Abbott TE, Larson D, Chen K. BreakDancer: identification of genomic structural variation from paired-end read mapping. In: Bateman A, Pearson WR, Stein LD, Stormo GD, Yates JR, editors. Current protocols in bioinformatics. Wiley; 2014. p. 15-6115611. https://doi.org/10.1002/0471250953.bi1506s45.

4. Korbel JO, Abyzov A, Mu X, Carriero N, Cayting P, Zhang Z, Snyder M, Gerstein MB. PEMer: a computational framework with simulation-based error models for inferring genomic structural variants from massive paired-end sequencing data. Genome Biol. 2009;10(2):23.

5. Gillet-Markowska A, Richard H, Fischer G, Lafontaine I. Ulysses: accurate detection of low-frequency structural variations in large insert-size sequencing libraries. Bioinformatics. 2015;31(6):801-8.

6. Zhang J, Wang J, Wu Y. An improved approach for accurate and efficient calling of structural variations with lowcoverage sequence data. BMC Bioinform. 2012;13 Suppl 6:6.

7. Trappe K, Emde A-K, Ehrlich H-C, Reinert K. Gustaf: Detecting and correctly classifying SVs in the NGS twilight zone. Bioinformatics (Oxford, England). 2014;30(24):3484-90.

8. Jiang Y, Wang Y, Brudno M. PRISM: pair-read informed split-read mapping for base-pair level detection of insertion, deletion and structural variants. Bioinformatics (Oxford, England). 2012;28(20):2576-83.

9. Abyzov A, Urban AE, Snyder M, Gerstein M. CNVnator: an approach to discover, genotype, and characterize typical and atypical CNVs from family and population genome sequencing. Genome Res. 2011;21(6):974-84.

10. Xie C, Tammi MT. CNV-seq, a new method to detect copy number variation using high-throughput sequencing. BMC Bioinform. 2009;10(1):80

11. Boeva V, Popova T, Lienard M, Toffoli S, Kamal M, Le Tourneau C, Gentien D, Servant N, Gestraud P, Rio Frio T, Hupé, P, Barillot E, Laes J-F. Multi-factor data normalization enables the detection of copy number aberrations in amplicon sequencing data. Bioinformatics. 2014;30(24):3443-50

12. Mareschal S, Ruminy P, Alcantara M, Villenet C, Figeac M, Dubois S, Bertrand P, Bouzelfen A, Viailly P-J, Penther D, Tilly $\mathrm{H}$, Bastard C, Jardin F. Application of the cghRA framework to the genomic characterization of Diffuse Large B-Cell Lymphoma. Bioinformatics (Oxford, England). 2017;33(19):2977-85.

13. Smith T, Heger A, Sudbery I. UMI-tools: modeling sequencing errors in Unique Molecular Identifiers to improve quantification accuracy. Genome Res. 2017:27(3):491-9.

14. Li H, Durbin R. Fast and accurate short read alignment with Burrows-Wheeler transform. Bioinformatics (Oxford, England). 2009;25(14):1754-60. 
15. Morgan M, Pagès H, Obenchain V, Hayden N. Rsamtools: binary alignment (BAM), FASTA, variant call (BCF), and tabix file import. Bioconductor version: Release (3.10); 2019. https://bioconductor.org/packages/Rsamtools/. Accessed 2019-12-04

16. Fraley C, Raftery AE, Scrucca L, Murphy TB, Fop M. mclust: Gaussian mixture modelling for model-based clustering, classification, and density estimation; 2019. https://CRAN.R-project.org/package=mclust. Accessed 2019-03-29.

17. Bengtsson H, Neuvial P, Seshan VE, Olshen AB, Spellman PT, Olshen RA. PSCBS: analysis of parent-specific DNA copy numbers; 2019. https://CRAN.R-project.org/package=PSCBS. Accessed 2019-12-04.

\section{Publisher's Note}

Springer Nature remains neutral with regard to jurisdictional claims in published maps and institutional affiliations.

- fast, convenient online submission

- thorough peer review by experienced researchers in your field

- rapid publication on acceptance

- support for research data, including large and complex data types

- gold Open Access which fosters wider collaboration and increased citations

- maximum visibility for your research: over 100M website views per year

At BMC, research is always in progress.

Learn more biomedcentral.com/submissions 